\title{
Generalized analysis of the deposition/splashing limit for one- and two-component droplet impacts upon thin films
}

\author{
Ronan Bernard ${ }^{* 1}$, Patrick Foltyn ${ }^{1}$, Anne Geppert ${ }^{1}$, Grazia Lamanna ${ }^{1}$, Bernhard Weigand ${ }^{1}$ \\ ${ }^{1}$ Institut für Thermodynamik der Luft- und Raumfahrt, Universität Stuttgart, Germany \\ *Corresponding author: ronan.bernard@itlr.uni-stuttgart.de
}

\begin{abstract}
Single drop impacts on thin liquid layers are of particular interest because of the ejection of secondary droplets, the so-called splashing. Only a few studies handle the deposition/splashing limit for two-component interaction, where the liquid properties of the impacting drop and wall film differ significantly.

This study aims at identifying a unified approach for one- and two-component interactions to determine the deposition/splashing limit. Therefore, a large database of both interactions is considered, which includes data from literature for one-component interactions plus the following binary combinations: hyspin-hexadecane, diesel-hexadecane and diesel-motor oil. Furthermore, a systematic study of two-component interactions with several silicon oils and hexadecane is performed. To map the outcomes, the Ohnesorge number $O h$ and the Reynolds number Re calculated with arithmetically averaged fluid properties between droplet and wall film fluid are chosen. The dimensionsless film thickness $\delta$ is added to form a $3 D$ plot, where one- and two-component experiments are combined.

Existing correlations from the literature are revised regarding both interactions and their consistency is checked. The investigated range of high viscosity fluids allow us to propose an improvement of the correlation for high $O h$. Our results show that the arithmetically averaged fluid properties lead to a good repartition of both one- and twocomponents interactions toward the deposition/splashing limit. They also corroborate the previous findings that an increase of $\delta$ inhibits splashing but its influence is decreasing with increasing $O h$.
\end{abstract}

\section{Keywords}

droplet impact, thin film, two-components interaction, splashing treshold.

\section{Introduction}

Single drop impact on a thin liquid layer (i.e. wall film) covers a wide range of industrial applications like coating, printing, cooling or combustion. They are of particular interest since they lead to the ejection of secondary droplets, the so-called splashing. The investigations concerning the droplet impact on wetted walls show that for a given Reynolds number $(R e)$, Ohnesorge number $(O h)$ and dimensionsless wall film thickness ( $\delta$, film thickness $h$ over droplet diameter $D$ ), the outcome of one-component droplet impact can be determined. Roughness of the wall can be neglected in the case of $0.1 \leq \delta \leq 1$ [9], when it is completely embedded in the liquid wall film. However, drop-film interactions are affected by the use of different fluids for the droplet and the liquid film. Very little is known for this two-component interaction: the available database is still sparse and therefore the validity of proposed correlations for the deposition/splashing limit cannot be yet generalized to any type of fluid combinations.

In this study, we consider a droplet impact of Newtonian fluids onto a wetted, cold, horizontal, solid surface. In the following, the denomination fluid1/fluid2 means that a fluid1 droplet impacts onto a fluid2 wall film and fluid1-fluid2 that both permutations were performed.

This study focuses on the deposition/splashing limit by incorporating both one- and two-component interactions into a single correlation. For this purpose, different existing correlations will be considered.

Many studies were performed for the deposition/splashing limit of one-component interaction such as Yarin and Weiss [7], Cossali et al. [2], Coghe et al. [8], Marengo and Tropea [9], Wang and Chen [11], Rioboo et al. [10], Vander Wal et al. [1] just to name a few.

The latter [1] encompasses a study on one-component interaction of twelve different fluids (ranging from alkanes to alcohols and including mixtures of glycerol-water). The dimensionsless parameters chosen to map the impacts outcome were $\mathrm{Oh}$ and $\mathrm{Re}$. The ranges of investigation were approximately [0.0026;0.017] and [800;14,000] for $\mathrm{Oh}$ and $R e$, respectively. The dimensionsless film thickness was kept constant and equal to 0.1 (obtained from the data $D=2 \mathrm{~mm}$ and $h=0.2 \mathrm{~mm}$ ). This restricts the validity of this correlation to $\delta=0.1$. The best fit of the data was found to be:

$$
\text { Oh } R e^{1.17}=63 \quad \text { (only valid for } \delta=0.1 \text { ). }
$$

Another well-known correlation is the one of Cossali et al. [2] that emphasizes the influence of the dimensionsless film thickness $\delta$ and of the dimensionsless roughness $R_{n d}=R_{a} / D$ on the onset of splashing. This correlation was obtained from experimental results with a mixture of water and glycerol, offering a quite wide predictive range. The traditional non-dimensionless numbers (based on single fluid properties) were $W e \in[200 ; 1600], O h \epsilon$ $[0.0022 ; 0.141]$ and $\delta \in[0.08 ; 1.2]$. The empirical correlation is based on the so-called impact factor $K=W e O h^{-0.4}$ inspired from a model on dry surfaces from Stow and Stainer [6]. In the case of thin film $\left(\delta \gg R_{n d}\right.$, to distinguish from very thin film), the influence of roughness can be neglected and the correlation for one-component interaction 

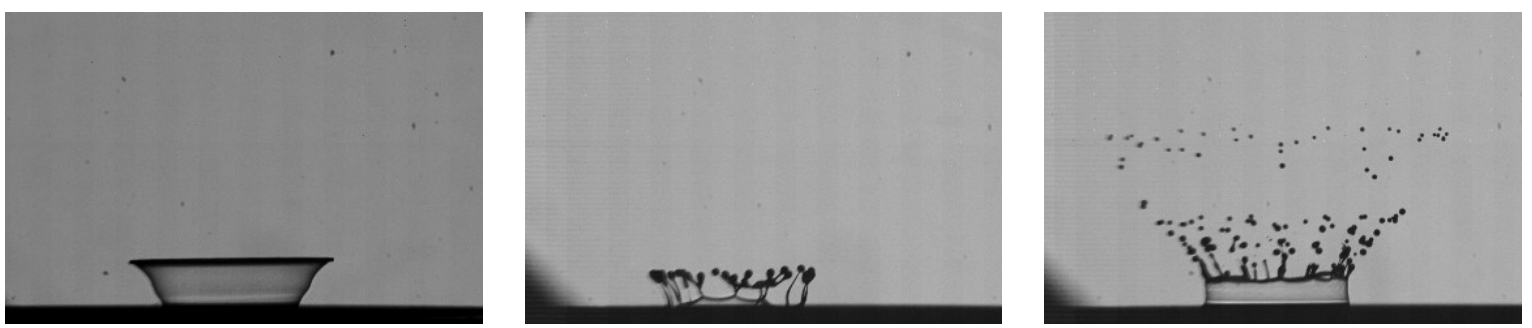

Figure 1. Classification of droplet impact outcomes: deposition (left), transition (middle) and splashing (right).

was found to be:

$$
W e O h^{-0.4}=2100+5880 \delta^{1.44}
$$

In [2], the splashing was defined as the formation of secondary droplets which has to be distinguished from deposition without secondary droplets. Cossali et al. [2] observed different outcomes for the same impact conditions near the deposition/splashing limit and so defined an upper and lower value of the critical $K$. Recently, Geppert et al. [3] introduced a regime, where a mixed impact behavior occurs. The latter could neither be distinctly assigned to splashing nor deposition, and was therefore classified as transition. In this study, we will also consider this transition state as a distinct outcome. The mapping is split into three regions, whose characteristic outcomes are depicted in Figure 1. On the right side, the splashing with the formation of liquid fingers along the upper lamella rim and the ejection of secondary droplets is depicted. On the left side, the deposition, where the crown originates from the wall film and then descends back to it without generating secondary droplets is shown. In between, the transition zone can be seen, where the crown is formed, secondary droplets are formed but not ejected and coalesce with the wall film.

Geppert et al. [3] also worked on a unified approach of the splashing/deposition limit for one- and two-component interaction. Their empirical correlation was based on the impact factor $K$ and $\delta$, similarly to Cossali et al. [2]. In [4], the authors noticed a major influence of the wall film fluid properties on the impact outcomes for two-component interaction (permutations of hyspin and hexadecane), that led to a different definition of the traditional dimensionsless parameters. They calculated the Ohnesorge number $O h_{v}$ with arithmetical averaged viscosities. The Weber number instead, was based on droplet properties since the kinetic energy is provided by the impacting drop and the surface tension of the two fluids did not differ significantly from each other. The resulting correlations for one- and two-component interactions were given as follow:

$$
\begin{aligned}
& W e^{0.625} O h_{v}^{-0.25}=114+145 \delta^{1.2} \\
& W e^{0.625} O h_{v}^{-0.25}=114+163 \delta^{1.2}
\end{aligned}
$$

The equations (3) and (4) are based on the hexadecane/hexadecane and hyspin-hexadecane interactions, respectively. This correlations are close to each other, and also close to the previous correlation of Cossali et al. [2]. Another study using even more averaged properties for two-component interaction was performed by Kittel et al. [5]. Based on experiments with silicon oils and water, they found that the conventional impact factor $K$ is not accurate for two-component interaction, because the wall film properties are not taken into account. They proposed a modified impact factor $K^{*}=R e_{v-B L}^{0.5} W e_{\sigma}$, where $v-B L$ and $\sigma$ indicate averaged viscosities based on the boundary layer theory and arithmetical averaged surface tensions, respectively. Plotting $K^{*}$ as a function of viscosity ratio between wall film and droplet fluids, Kittel et al. [5] reported that a tendency of splashing treshold can be seen, unlike the conventional impact factor $K$. This shows that both properties of the droplet and the wall film have to be taken into account. In this study, all properties (viscosities, surface tensions and densities) will be arithmetically averaged, leading to the following dimensionsless parameters:

$$
W e_{\text {ave }}=\frac{\left(\rho_{f}+\rho_{d}\right) V^{2} D}{\sigma_{f}+\sigma_{d}}, \quad O h_{\text {ave }}=\frac{\nu_{f}+\nu_{d}}{2} \sqrt{\frac{\rho_{f}+\rho_{d}}{D\left(\sigma_{f}+\sigma_{d}\right)}}, \quad R e_{\text {ave }}=\frac{2 V \cdot D}{\nu_{f}+\nu_{d}}
$$

with the density $\rho$, the kinematic viscosity $\nu$, the surface tension $\sigma$, the impact velocity $V$ and the droplet diameter $D$. The subscripts $f$ and $d$ denote film and droplet. The arithmetical average allows to take both, droplet and film fluids, into account for two-component interactions, and has no influence in case of one-component interaction. Therefore, it allows the use of the previous correlations from the literature.

The purpose of this study is to propose an unified approach for the correlation of the deposition/splashing limit for one- as well as two-component interactions. This can be attained with the use of arithmetically averaged parameters in the conventional dimensionsless numbers. The governing parameters will be put together to offer an overall view of their influence. The existing correlations are put into perspective and their consistency will be checked. To achieve these goals, a large database is needed. Therefore, a systematic study of two-component interactions 
was performed by including variations in viscosities, surface tensions and densities. Specifically, we permutated several silicon oils and hexadecane for the droplet and wall film liquids. Furthermore, a large database of one- and two-component interactions from [1, 2, 3] is combined with the performed experimental results.

\section{Material and methods \\ Material}

The experimental setup used in this work is the same as the one used by Geppert et al. [4]. It consists of three main systems: the dropper, the impingement area and a two-perspectives shadowgraphy imaging system.

The droplets are generated with a pump, which delivers the fluid from the reservoir to a needle having a fixed diameter of $0.8 \mathrm{~mm}$. The flow rate is low enough to make the droplet drip off by gravity and then take advantage of the gravitational acceleration. The impact velocity is adjusted with the height of the needle.

During its fall, the droplet is passing through a laser light barrier that triggers the high-speed camera (Photron Fastcam SA1.1). The imaging system allows the simultaneous recording of a frontal and lateral view making the observation of non-axisymmetric features during the impact possible. The target zone is constantly back-lighted by two photodiodes. The images are then redirected by a set of mirrors and both light paths are focused by a prime lens on the camera sensor $(896 \times 320$ pixels $)$. The frame rate of the camera and the shutter speed were set to $20,000 \mathrm{fps}$ and 1/92,000 s, respectively. The magnification of the optical set is $1: 4$ to allow the observation of the entire impact area.

The impact area consists of a smooth sapphire glass plate on which a thin metallic ring (diameter $60 \mathrm{~mm}$, height $0.6 \mathrm{~mm}$ ) is glued. The inner region of the ring is filled with the wall film fluid till the desired thickness is reached. The measurement of the wall film thickness is realized with a commercial device (Micro-Epsilon - Model, IFC2451 controller and IFS2405-3 sensor) using the confocal chromatic imaging (CCl) technique, a non-intrusive distance measurement method. This method uses the difference in refractive index between the wall film fluid and the plate analyzing the spectral distribution of the reflected light. More details about the experimental setup can be found in [3].

\section{Method}

A systematic study of two-component interaction is carried out where the properties of the test liquids was varied. Specifically, we interchanged silicon oils with different viscosities and hexadecane for the droplet and wall film liquid, respectively. Fluid properties are listed in table 1 . The viscosity is doubled each time for $B 5, B 10$ and $B 20$.

Table 1. Fluid properties at ambient temperature and pressure.

\begin{tabular}{c|ccc}
\hline & density $\left[\mathrm{kg} \cdot \mathrm{m}^{-3}\right]$ & surface tension $\left[10^{-3} \mathrm{~N} \cdot \mathrm{m}^{-1}\right]$ & viscosity $\left[10^{-6} \mathrm{~m}^{2} \cdot \mathrm{s}^{-1}\right]$ \\
\hline Hexadecane & 773 & 27.5 & 4.46 \\
B3 & 900 & 18.0 & 3.0 \\
B5 & 920 & 19.2 & 5.0 \\
B10 & 945 & 20.2 & 10.0 \\
B20 & 955 & 20.6 & 20.0
\end{tabular}

The wall film thickness was adjusted to systematically achieve a dimensionsless film thickness of approximately $\delta=0.11$, depending on the droplet diameter. The droplet diameter is related to the constant needle diameter and the fluid properties. The averaged droplet diameters are roughly $1.9 \mathrm{~mm}$ for silicon oils and $2.4 \mathrm{~mm}$ for hexadecane. The free fall height was equal to $1.28 \mathrm{~m}$ and $0.87 \mathrm{~m}$, resulting in an impact velocity of approximately $4.3 \mathrm{~m} / \mathrm{s}$ and $3.6 \mathrm{~m} / \mathrm{s}$, respectively. For each test condition, a set of at least three experiments was performed, ensuring reproducibility and reducing measurement errors. The experimental procedure was carried out as follow: the dropper was installed for a specific droplet fluid. A series of experiments was performed with another fluid as wall film. Before each experiment, the pool was cleaned with ethanol and refilled. The wall film fluid was progressively added to the pool depending on the measured wall film thickness. The thickness was measured after a specific delay time to ensure the film liquid at rest and averaged over 10,000 values.

After all fluids were tested as wall film, the entire dropper (pipes and needle) was replaced for the next droplet fluid. The experimental uncertainties for this experimental setup are detailed in [3]. The measurement of droplet diameter and impact velocities are performed with an in house MATLAB routine from consecutive images just before impact. The measurement accuracy for the droplet diameter and the impact velocity is in the range of $2,1 \%$ and $2 \%$, respectively. The error related to the measurement of the film thickness is less than $1 \%$ (which is also reported in Geppert et al. [3]). The classification of the outcomes (splashing, transition or deposition) was judged visually for each experiment.

To enlarge the database, other experimental results from Geppert et al. [4, 3] were added, performed on the same experimental setup for the interactions hexadecane/hexadecane and hyspin-hexadecane. In addition, previous unpublished experimental results were also added for the interactions hyspin/hyspin, diesel/diesel, diesel/Castrol 5W-30 and diesel/hexadecane. Fluid properties are listed in Table 2. The investigations with these additional combinations were performed for a wide range of film thicknesses and Weber number.

Overall, the entire database from this experimental setup was given by: $\operatorname{Re}_{\text {ave }} \in[98 ; 3654] ; O h_{\text {ave }} \in[0.012 ; 0.283]$; $\delta \in[0.03 ; 0.55]$ and contains more than 2000 experiments. 
Table 2. Additional fluid properties at ambient temperature and pressure.

\begin{tabular}{c|ccc}
\hline & density $\left[\mathrm{kg} \cdot \mathrm{m}^{-3}\right]$ & surface tension $\left[10^{-3} \mathrm{~N} \cdot \mathrm{m}^{-1}\right]$ & viscosity $\left[10^{-6} \mathrm{~m}^{2} \cdot \mathrm{s}^{-1}\right]$ \\
\hline Hyspin & 878 & 28.7 & 18.0 \\
Diesel & 827 & 28.0 & 4.0 \\
Castrol 5W-30 & 846 & 31.0 & 156.2
\end{tabular}

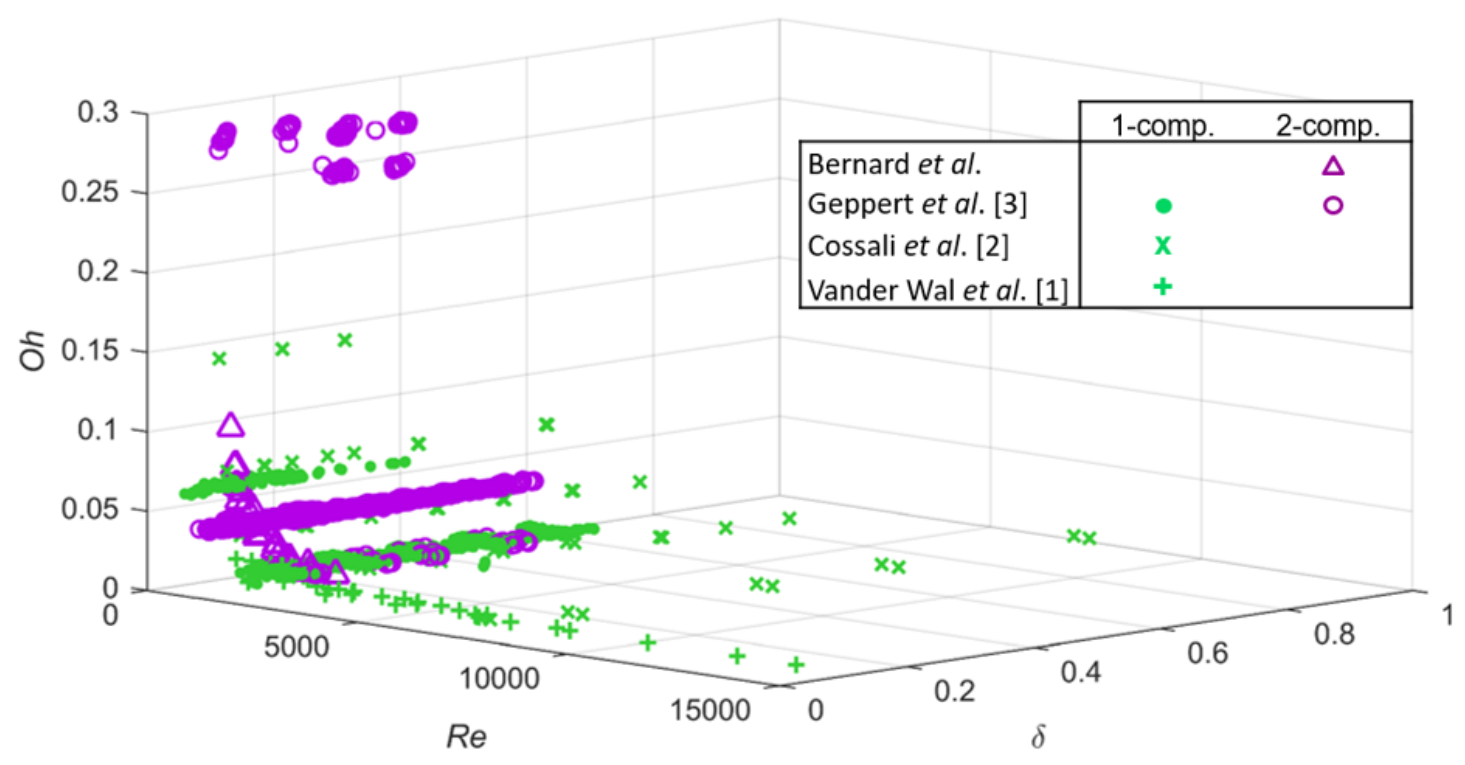

Figure 2. Common mapping of the investigated range of one- and two-components interactions (green and purple symbols, respectively) in $\left(O h_{\text {ave }}, R e_{\text {ave }}, \delta\right)$ from the referenced sources.

\section{Results and discussion}

To map the different outcomes, we follow the approach of Vander Wal et al. and use arithmetically averaged properties, meaning that $O h_{\text {ave }}$ and $R e_{\text {ave }}$ are chosen as defined in equation 5. Using the third governing parameter $\delta$, a $3 D$ plot containing the entire database is created and shown in Figure 2.

Each point represents one experiment from the considered authors, putting the investigated ranges for one- and two-component interactions into perspective (green and purples symbols, respectively). The dots and circles are the experiments from [3], where each array corresponds to a detailed investigation for a given combination. Similarly, the data from Cossali et al. [2] are plotted with green crosses. The data from Vander Wal et al. [1] varying the fluid properties are distributed along $\delta=0.1$ in green plus signs. Finally, the sets of two-component experiments of this work are drawn in purple triangles, completing the investigated range of [1] and filling the gaps between the steps experiments of [3].

Several slices in Figure 2 will be considered to enhance the influence of each parameter separatly and compare correlations. In the following figures, each symbol represents a particular fluid combination, as summarized in Table 3. The red symbols belong to the splashing state, the blue ones to deposition and the green ones were classified to be in the transition state, as defined in the introduction.

The chosen reference correlations are the ones of Vander Wal et al. [1] and Cossali et al. [2], expressed in equations (1) and (2), respectively. Given that $O h, R e$ and $W e$ are linked by $O h=\sqrt{W e} / R e$, these correlations are equivalent and can be compared.

First, we focus on the slice $\delta=0.1$ where the Vander Wal correlation [1] is valid. We consider the range $\delta \in[0.1 ; 0.12]$, which contains the series of two-component experiments of silicon oils and hexadecane. The slice is shown in Figure 3 with the legend reference given in Table 3.

The yellow dashed line represents the correlation of Vander Wal et al. [1]. It gives a good trend for the deposition/splashing limit, but seems to lie below what could be seen as the best fit. This is particularly the case for Re smaller than 1000, which also corresponds to the lower limit of the investigated range by Vander Wal et al. [1] (roughly $R e \in[800 ; 14,000]$ ). In fact, the correlation fits well for higher Re. The purple dashed line corresponds to the correlation of Cossali et al. [2] adapted to the formalism of Vander Wal et al. [1]. Both correlations show high consistency with each other. Similarly, the correlation of Geppert et al. [3] based on the interaction hyspin/hexadecane - equation (4) with averaged viscosities only - is drawn as dashed cyan line in Figure 3. Both are very close together, as shown in [3], highlighting the similarity of their approaches.

All these correlations show the same asymptotical behaviour at high $R e$ but differ for $R e<1000$. In fact, the different exponents on $R e$ in the correlations change the curvature for a similar asymptotical behaviour. A higher exponent (e.g. Cossali vs Vander Wal) leads to an increase of the deposition/splashing limit. 
Table 3. Legend of fluid combinations and droplet impact outcomes for Figures 3, 4, 6.

\begin{tabular}{c|c}
\hline & Symbol \\
\hline Hyspin-Hexadecane & $\circ$ \\
Hexadecane/Hexadecane & $\times$ \\
Hyspin/Hyspin & $\star$ \\
Diesel/Castrol 5W30 & $\cdot$ \\
Diesel/Diesel & $\square$ \\
Diesel/Hexadecane & $\diamond$ \\
Oil-Oil & $\triangle$ \\
Oil-Hexadecane & $\nabla$ \\
Cossali et al. experiments & + \\
Vander Wal et al. experiments & \multirow{2}{}{} \\
blue & deposition \\
green & transition \\
red & splashing
\end{tabular}

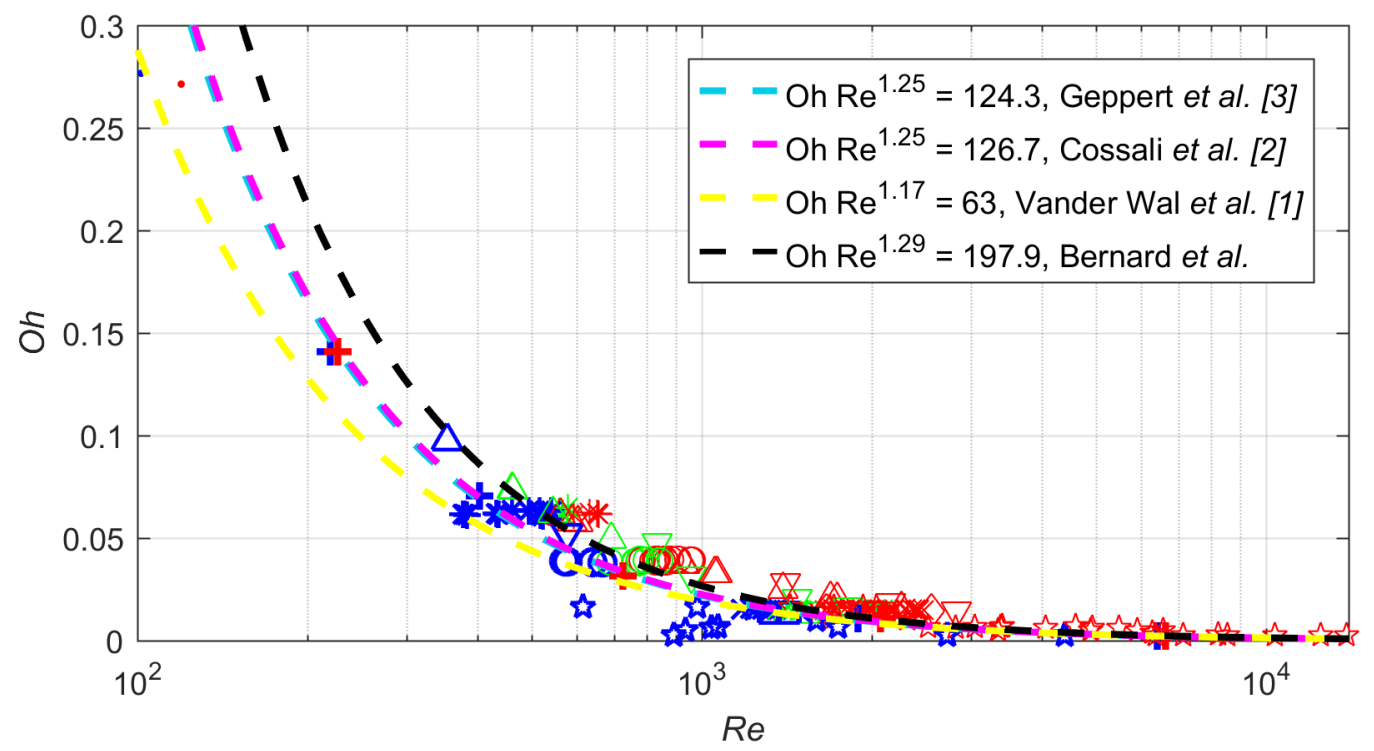

Figure 3. Mapping of droplet impact outcomes for $\delta=0.1$ on a logarithmic scale. Comparison of the correlations of Vander Wal et al. [1] (yellow dashed line), Cossali et al. [2] (purple dashed line), Geppert et al. [3] (cyan dashed line) and from equation (7) (black dashed line). Legend referenced in Table 3.

The experiments performed with high $\mathrm{Oh}$ apparently show an even higher deposition/splashing limit in the range of $R e<1000$. To better fit the transition zone in this range, the following correlation, drawn as a black dashed line in Figure 3, can be used:

$$
\text { Oh } R e^{1.29}=197.9 \quad \text { (only valid for } \delta=0.1 \text { ). }
$$

Note that the two-component interactions (Hyspin-Hexadecane $\circ$, Diesel/Hexadecane $\diamond$, Oil-Oil $\triangle$, Oil-Hexadecane $\nabla$ ) fit the correlation (6), as well as the one-component interactions (Hexadecane/Hexadecane $\times$, Hyspin/Hyspin $\star$, Diesel/Diesel $\square$ ), despite significant differences in viscosity, surface tension and density. This supports the use of averaged properties to take into account both droplet and wall film fluids. The correlation (6), expressed for $\delta=0.1$ offers the same behaviour as the previous correlations from literature for high $R e$. For low $R e$, the deposition/splashing limit was adjusted to be higher.

The influence of the wall film thickness will be now considered in the top-view of the Figure 2, presenting $R e$ as function of $\delta$ for several values of $O h$ in Figure 4. To distinguish between the several steps of Ohnesorge numbers, vertical dashed lines are drawn. The arrow indicates increasing $O h$. The domain on the right side contains mostly points from the slice $O h=0.015$, the middle one from $O h=0.04$ and the left one from all points where $O h$ is higher than 0.14 (correlations are drawn with $O h=0.141$ and $O h=0.25$ ). The interactions hyspin/hyspin $(\star)$, oil-oil $(\triangle)$, oil-hexadecane $(\nabla)$ as well as the data from Cossali et al. [2] and Vander Wal et al. [1] were removed to avoid overlapping (except for $O h=0.141)$. 


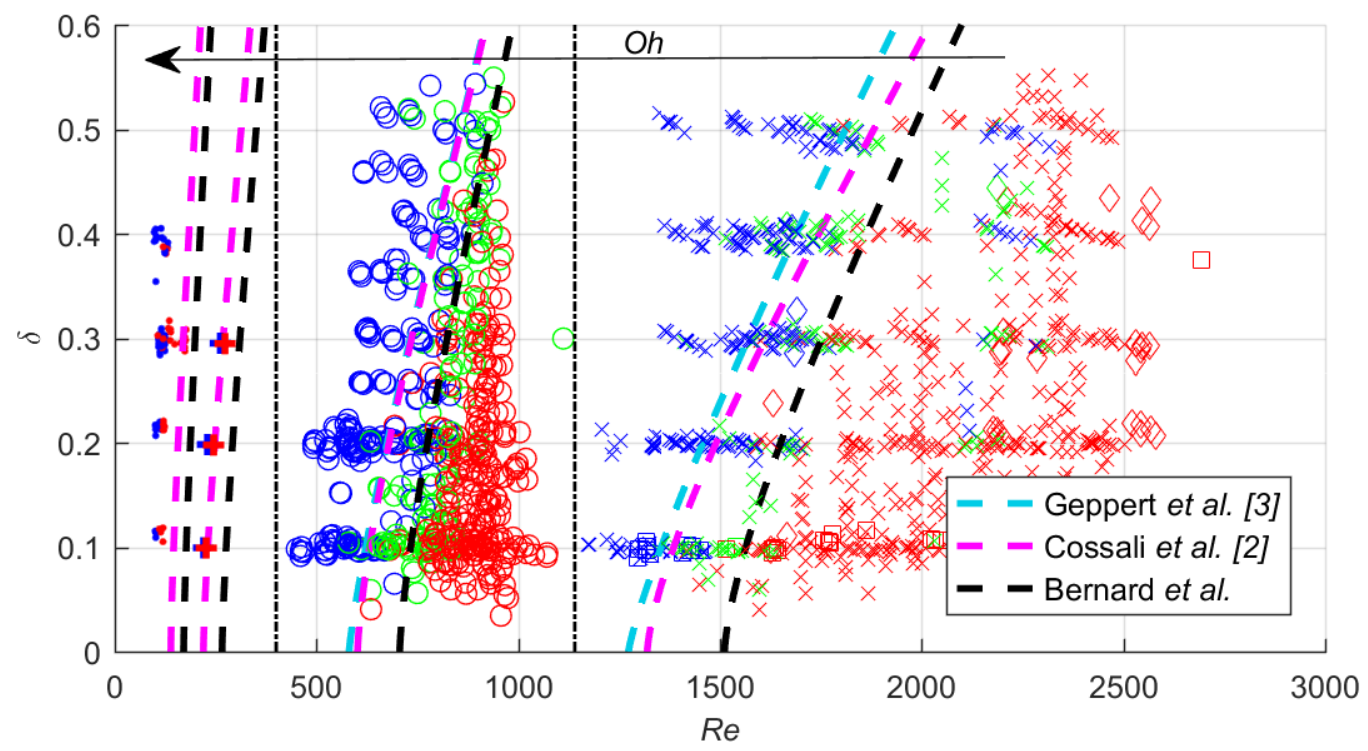

Figure 4. Influence of $\delta$ on the deposition/splashing limits for $O h=0.015$ (right), $O h=0.04$ (middle), $O h=0.141$ and $O h=0.25$ (left). Legend referenced in Table 3.
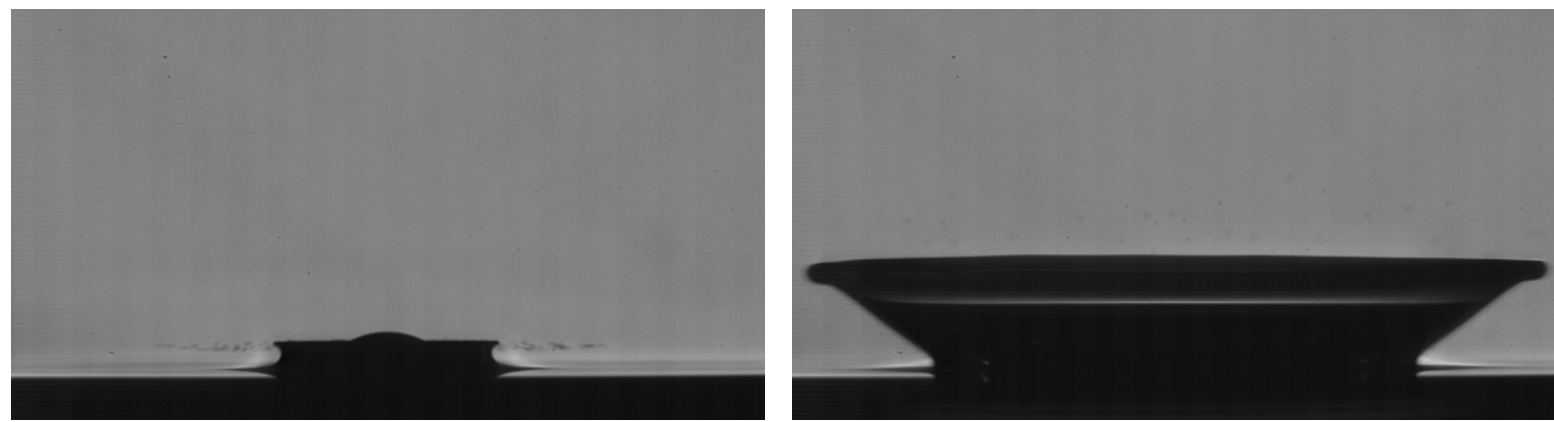

Figure 5. Impact of a diesel droplet onto a Castrol 5W-30 wall film. Left: ejection of tiny droplets at the beginning of the impact. Right: typical deposition morphology of the crown just before receding

For each domain, the correlation of Cossali et al. [2] is drawn as dashed purple line. The adjusted correlations of Geppert et al. [3] for hexadecane/hexadecane $(O h=0.015)$, equation (3), and hyspin-hexadecane $(O h=0.04)$, equation (4), are also plotted in their validity range as cyan dashed lines. In each domain, these correlations are very close together, both in the transition zone and comprised in the experimental uncertainties, see [3]. The dark dashed correlations in Figure 4 corresponds to the one expressed previously for $\delta=0.1$ in equation (6), with a dependency on $\delta$ adapted from the correlation of Cossali et al. [2]. The best fit, consistent with equation (6), was found to be:

$$
\text { Oh } \operatorname{Re}^{1.29}=\left(4400+8900 \delta^{1.44}\right)^{\frac{1}{1.6}}
$$

Once again, it is located close to the other correlations, in the transition zone. However, one can observe that for high $O h$ (left domain), the splashing/deposition limit seems to be much higher than the real one, where red dots (splashing of Diesel/Castrol 5W30) can be seen on the left side of the correlation. Considering the very high viscosity of the employed wall film, and also the high viscosity ratio between droplet and film, the classification of this outcomes as splashing can be discussed: the ejection of tiny droplets at the very beginning of the impact can be observed (Figure 5, left) similar to prompt splashing, explaining the classification. But considering now the crown wall that follows (Figure 5, right) no droplets are formed and this feature is closer to deposition. This explains also that no transition state is observed in this domain. Further investigations have to be performed to draw a conclusion in this domain of high viscosity fluids.

Considering the influence of $\delta$ on the deposition/splashing limit, one can easily see in Figure 4 that the required $R e$ to onset splashing are increasing with increasing $\delta$. In other words, an increase of $\delta$ inhibits splashing, as reported in literature [2, 3]. However, the deposition/splashing limits are becoming straighter with increasing $O h$, as one can see in the middle and left domain in Figure 4, meaning that the influence of $\delta$ on the deposition/splashing limit is decreasing. 
All these effects are now summarized by defining a deposition/splashing limit surface, depicted in Figure 6 . The equation of this surface, expressed in equation (7), is made of the correlation deducted in slice $\delta=0.1$ for the left side and of the adapted dependency of $\delta$ inspired from Cossali et al. on the right side. This surface, plotted in Figure 6, splits the $3 D$ domain in two parts: above the surface (top view, left) are the splashing outcomes -red symbols- and below the surface (bottom view, right) are the deposition outcomes -blue symbols-. The transition outcomes mostly overlap the surface limit.
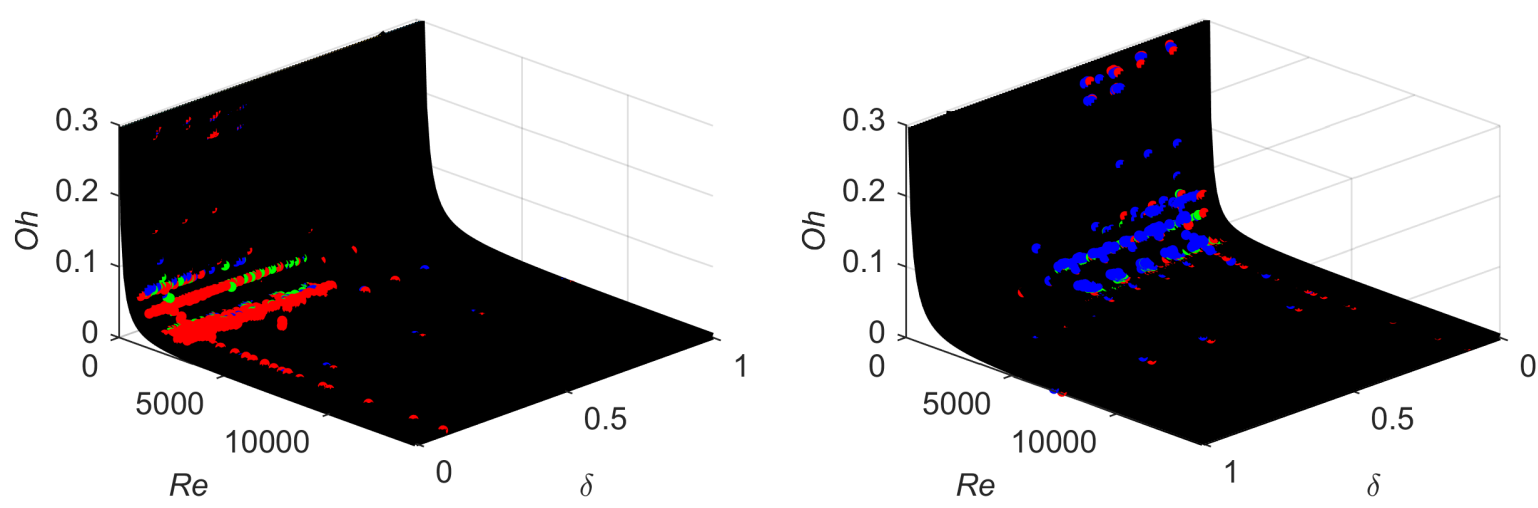

Figure 6. Mapping of the outcomes split by the deposition/splashing surface limit defined in equation (7), view from the top -splashing- (left) and from the bottom -deposition- (right). Legend referenced in Table 3.

Considering the available database, 1660 outcomes over 2197 experiments are on the correct side of the deposition/splashing surface limit, which represents a accuracy of $75.6 \%$. Transition outcomes can be in both deposition and splashing regions. For comparison, the surface based on the correlation of Cossali et al. [2] would bring an accuracy of $72.7 \%$. This improvement is due to the domain for $O h$ larger than 0.025 , where the curvature of the limit seemed to be underestimated. However, more experiments in this range have to be performed. For lower $O h$, the surface limit stays consistent with the previous correlations from literature $[1,2,3]$. Furthermore, the arithmetically averaged properties lead to a good repartition toward the limit, which makes this correlation valid also for two-component interaction.

\title{
Conclusion
}

The deposition/splashing limit is of particular interest for single drop impacts upon thin liquid layers due to the ejection of secondary droplets. Many studies investigated this limit for one-component interactions, but only a few focused on two-component splashing, where the two liquids differ significantly.

In this study, an unified approach of the deposition/splashing limit for both one- and two-component interactions is investigated. A large database is used: for one-component, the results from Cossali et al. [2] and Vander Wal et al. [1] are combined. Furthermore, experiments from Geppert et al. [3, 4] for one- and two-component interactions of hyspin and hexadecane, are added, plus the combinations of diesel-hexadecane and diesel-motor oil. Lastly, a systematic study of two-component interactions with several silicon oils and hexadecane is performed.

To map the outcomes, the Ohnesorge number $O h$ and the Reynolds number Re are chosen. These are calculated with arithmetically averaged properties between droplet and wall film fluid. Besides, the dimensionsless film thickness $\delta$ is added to form a 3D plot where one- and two-component experiments are combined.

An analyse of the mapping shows that the arithmetically averaged properties lead to a good repartition of the one- and two-component outcomes toward the deposition/splashing limit, despite significant differences in the fluid properties of the combinations.

This study enhances also that all previous reported correlations (Vander Wal et al. [1], Cossali et al. [2] and Geppert et al. [3]) show a high consistency among each other, within the experimental uncertainty.

Since the database includes high viscous fluids, it was possible to propose an improved correlation for the deposition/splashing limit in the range of low $\operatorname{Re}(\operatorname{Re}<1000)$.

\section{Acknowledgements}

The authors kindly acknowledge the financial support of this work by the Deutsche Forschungsgemeinschaft (DFG) in the frame of the International Research Training Group "Droplet Interaction Technologies" (DROPIT).

\author{
Nomenclature \\ Greek symbols \\ $\rho \quad$ density $\left[\mathrm{kgm}^{-3}\right]$ \\ $\nu \quad$ kinematic viscosity $\left[\mathrm{m}^{2} \mathrm{~s}^{-1}\right]$
}




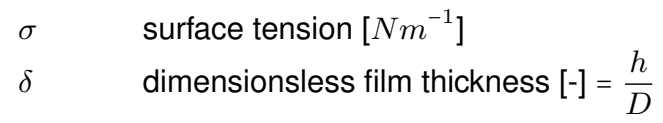

Latin symbols

$V \quad$ impact velocity $\left[\mathrm{m} \mathrm{s}^{-1}\right]$

$D \quad$ droplet diameter [m]

$h \quad$ film thickness [m]

Re Reynolds number [-] $=\frac{V D}{\nu}$

Oh Ohnesorge number [-] $=\nu \sqrt{\frac{\rho}{\sigma D}}$

$W e \quad$ Weber number [-] $=\frac{\rho V^{2} D}{\sigma}$

$K \quad$ impact factor [-] $=$ We $\stackrel{\sigma}{O} h^{-0.4}$

$K^{*} \quad$ impact factor from [5] with averaged viscosities and surface tensions [-]

$R_{a} \quad$ arithmetic averaged roughness

Subscripts

$d \quad$ droplet

$f \quad$ wall film

$v \quad$ averaged viscosities

$v-B L$ averaged viscosities based on boundary layer theory

$\sigma \quad$ averaged surface tensions

ave all properties averaged

\section{References}

[1] Vander Wal, R. L., Berger, G. M., \& Mozes, S. D. (2006). The splash/non-splash boundary upon a dry surface and thin fluid film. Experiments in fluids, 40(1), 53-59.

[2] Cossali, G. E., Coghe, A., \& Marengo, M. (1997). The impact of a single drop on a wetted solid surface. Experiments in Fluids, 22(6), 463-472.

[3] Geppert, A., Chatzianagnostou, D., Meister, C., Gomaa, H., Lamanna, G., \& Weigand, B. (2016). Classification of Impact Morphology and Splashing/Deposition Limit for n-Hexadecane. Atomization and Sprays, 26(10), 9831007.

[4] Geppert, A., Gomaa, H., Meister, C., Lamanna, G., \& Weigand, B. (2014). Droplet wall-film interaction: impact morphology and splashing/deposition boundary of hyspin/n-hexadecane two-component systems. In Proceedings of the 26th Annual Conference on Liquid Atomization and Spray Systems, ILASS-Americas.

[5] Kittel, H.M., Roisman, I.V., Tropea, C. (2016). Outcome of Drop Impact onto a Liquid Film of Different Viscosities. In Proceedings of the 27th Annual Conference on Liquid Atomization and Spray Systems, ILASS-Europe.

[6] Stow, C. D., \& Stainer, R. D. (1977). The physical products of a splashing water drop. Meteorological Society of Japan Journal, 55, 518-532.

[7] Yarin, A. L., \& Weiss, D. A. (1995). Impact of drops on solid surfaces: self-similar capillary waves, and splashing as a new type of kinematic discontinuity. Journal of Fluid Mechanics, 283, 141-173.

[8] Coghe, A., Brunello, G., Cossali, G. E., Marengo, M., \& TeMPE-CNR, M. I. (1999). Single drop splash on thin film: measurements of crown characteristics. In ILASS Europe.

[9] Tropea, C., \& Marengo, M. (1999). The impact of drops on walls and films. Multiphase Science and Technology, 11(1), 19-36.

[10] Rioboo, R., Bauthier, C., Conti, J., Voue, M., \& De Coninck, J. (2003). Experimental investigation of splash and crown formation during single drop impact on wetted surfaces. Experiments in fluids, 35(6), 648-652.

[11] Wang, A. B., \& Chen, C. C. (2000). Splashing impact of a single drop onto very thin liquid films. Physics of fluids, 12(9), 2155-2158. 